\title{
EFFECTS OF TRANSCRANIAL DIRECT CURRENT STIMULATION (tDCS) ON CORTICAL ACTIVITY: A COMPUTATIONAL MODELING STUDY
}

\author{
B. Molaee-Ardekani1 ${ }^{1,2}$, J. Márquez-Ruiz $^{3}$, I. Merlet ${ }^{1,2}$, R. Leal-Campanario ${ }^{3}$, A. Gruart $^{3}$, R. Sánchez- \\ Campusano $^{3}$, G. Birot ${ }^{1,2}$, G. Ruffini ${ }^{4}$, J.M. Delgado-García ${ }^{3}$ and F. Wendling, \\ ${ }^{1}$ INSERM, U642, Rennes, F-35000, France \\ ${ }^{2}$ Université de Rennes 1, LTSI, F-35000, France \\ ${ }^{3}$ University Pablo de Olavide, Sevilla, Spain \\ ${ }^{4}$ Starlab, Barcelona, Spain
}

Abstract

Although it is well-admitted that transcranial Direct Current Stimulation (tDCS) allows for interacting with brain endogenous rhythms, the exact mechanisms by which externally-applied fields modulate the activity of neurons remain elusive. In this study a novel computational model (a neural mass model including subpopulations of pyramidal cells and inhibitory interneurons mediating synaptic currents with either slow or fast kinetics) of the cerebral cortex was elaborated to investigate the local effects of tDCS on neuronal populations based on an in-vivo experimental study. Model parameters were adjusted to reproduce evoked potentials (EPs) recorded from the somatosensory cortex of the rabbit in response to air-puffs applied on the whiskers. EPs were simulated under control condition (no tDCS) as well as under anodal and cathodal tDCS fields. Results first revealed that a feed-forward inhibition mechanism must be included in the model for accurate simulation of actual EPs (peaks and latencies). Interestingly, results revealed that externally-applied fields are also likely to affect interneurons. Indeed, when interneurons get polarized then the characteristics of simulated EPs become closer to those of real EPs. In particular, under anodal tDCS condition, more realistic EPs could be obtained when pyramidal cells were depolarized and, simultaneously, slow (resp. fast) interneurons became de- (resp. hyper-) polarized. Geometrical characteristics of interneurons might provide some explanations for this effect. 


\section{Introduction}

Over the past decade, the use of transcranial Current Stimulation tCS (either direct tDCS or alternating tACS) has considerably increased both in clinical and research studies as this noninvasive method was shown to modulate the activity and performance of the brain [1-3]. As an example, some recent studies [4] showed that anodal tDCS during sleep can increase the retention of word pairs. Although many cognitive/clinical applications of tCS have been investigated over the two past decades [5], the exact mechanisms by which externally-applied fields influence the activity of neuronal populations located in the cerebral cortex are not well described yet [6]. Better understanding the impact of currents induced by tCS on neuronal systems is fundamental as it may lead to substantial improvement of stimulation devices and protocols, for both diagnostic and therapeutic purposes. Several in-vitro and in-vivo studies have been conducted to address such issues by exploring the behavior of small and large networks of neurons under the effect of electric or magnetic stimulations (see $[7,8]$ among others). These studies constitute first attempts to address some fundamental questions about the role of stimulation parameters (stimulation intensity, duration, frequency, repetition, position/orientation/shape of stimulating devices) and subsequent effects on stimulated systems.

More recently, biophysicists have also proposed some computational models aiming to provide insight into the mechanisms involved in the interaction between neurons and externally-applied fields. Most of these models account for the effects of external fields on single neurons [9-12] and very few models have been designed to investigate the behavior of networks of neurons under the effect of applied fields $[13,14]$. 
In this context, the objective of this study is to analyze in detail how a neuronal assembly is affected by the electric field, and how its response, as observed in local field potentials (LFPs), relates to the applied electric field parameters. For this purpose, we have elaborated a macroscopic neurophysiologically-relevant computational model of the cerebral cortex. This model is used to thoroughly investigate and explain the immediate effects of applied electric field on evoked potentials (EPs), as recorded in the somatosensory cortex (SSC) of the rabbit.

\section{Materials and methods}

\subsection{Experimental model and in-vivo recordings}

In order to experimentally assess the influence of tDCS on local field potentials generated in the cerebral cortex, three male rabbits were prepared for chronic intracortical recording from the SSC. In short, and following procedures described elsewhere [15], the animal's skull was drilled through the parietal bone centered on the right S1 vibrissa area (row $\mathrm{C}$, anterioposterior $(\mathrm{AP})=-1.7 \mathrm{~mm}$, lateral $(\mathrm{L})=7 \mathrm{~mm}[16])$ and four silver ball stimulating electrodes ( $1 \mathrm{~mm}$ in diameter) were symmetrically placed around the drilled window over the skull, under deep anesthesia.

A head-holding system was used to cement the stimulating electrodes to the skull which were then placed into a socket. After 1 week allowance for recovery, animals were habituated to the restraining box for about three days. Once habituated to the recording system, a glass micropipette $(0.5-1.5 \mathrm{M} \Omega)$ was inserted into the SSC corresponding to the whiskers for acquisition of LFPs. In order to avoid as much as possible the variability of the location of the micropipette with respect to the stimulating electrodes, the diameter of the recording area was kept small $(2 \mathrm{~mm})$ and the micropipette was located at its centre equidistant to the four silver ball electrodes. The final recording position of the micropipette (usually, $1.2-1.6 \mathrm{~mm}$ depth w.r.t. cortical surface) was determined by mapping the receptive field of the contra-lateral 
whisker pad by the presentation of air-puffs to the whiskers. Once the LFP specificity was established for a particular whisker pad region, air pulses $\left(100 \mathrm{~ms}, 2 \mathrm{Kg} / \mathrm{cm}^{2}\right)$ were delivered every $10 \pm 3 \mathrm{~s}$ before (control) and during application of tDCS (anodal or cathodal current). It is noteworthy that, to ease further comparisons, EPs were always recorded with the same microelectrode penetration such that differences in waveforms cannot be attributed to the electrode depth.

tDCS was applied to the four silver ball electrodes, whilst a saline-soaked sponge $(5 \times 7 \mathrm{~cm})$ attached to the ear contra-lateral to the recording side served as the counter electrode. This experimental design permitted to record intracortical somatosensory evoked potentials (SEPs) inside the cortical region affected by DC currents. The four silver balls, placed equidistantly with respect to the recording place, offered us the possibility to directly measure neuronal population cortical responses to sensory input under simultaneously-applied DC fields. Anodal and cathodal stimulations were performed with current intensities equal to +1 and $1 \mathrm{~mA}$, respectively. Although our experimental design does not allow to estimate the current density $(\mathrm{mA} / \mathrm{cm} 2)$ applied to the animal cortex, we experimentally observed that a $1 \mathrm{~mA}$ applied current affected the neuronal activity (reduction or enlargement of SEPs). Evoked potentials in response to air-puffs were recorded (sampling frequency $1.66 \mathrm{KHz}$ ) for a few minutes under anodal and cathodal currents, as well as, under no stimulus (control) condition for further analyses.

\subsection{Computational model}

Detailed (microscopic) and lumped-parameter (mesoscopic) models have been proposed to describe neuronal systems involved in the generation of brain activity $[17,18]$. In this study, the mesoscopic level was chosen to describe the "average" behavior of a population of neuron influenced by an externally-applied electric field. The basic idea is to capture, in a relatively 
simple representation, the main features of subpopulations of neurons (typically pyramidal cells and interneurons) and their interactions (mostly synaptic). This class of models (also referred to as "neural mass") is appropriate to describe the temporal activity of a local neuronal population (no space variable). If needed, it may be extended to the class of meanfield models (both time and space variables) [19]. Indeed, this extension can be easier achieved compared to a detailed (i.e. cellular level) model. Therefore, having a neural mass model which accounts for the effect of tDCS is the first step toward a more complete model describing how a larger brain region, and even a network involving several regions, may respond to this type of stimulation. Following this approach, we designed a computational model aimed at reproducing, sensory evoked potentials, locally recorded from the SSC of the rabbit under tDCS effect, as described in section 2.1.

\subsubsection{Mesoscopic model of the somatosensory cortex}

The "global" architecture of the model is similar to that of the mesoscopic-level model recently proposed in [20]. The novelty stems from the fact that we specifically introduced for the purpose of this study i) a feed-forward inhibition mechanism [21, 22] and ii) a representation of the tDCS effect.

The model is composed of different subpopulations of cells (principal pyramidal cells and interneurons) which interact via excitatory and inhibitory synaptic connections. The model accounts for the main types of cells present in the cerebral cortex:

- Pyramidal cells which are identified as type $P$ sub-population in the model.

- Axon-, soma- and proximal dendrite-targeting cells such as basket cells and chandelier cells. These cells are gathered together into the same subpopulation (identified as type I interneurons,) since they mediate $\mathrm{GABA}_{\mathrm{A}, \text { fast }}$ currents on type $P$ cells. 
- Dendrite-targeting cells such as bitufted, bipolar and double bouquet cells. These cells are gathered also together into the same subpopulation (identified as type $I^{\prime}$ interneurons) since, in this case, they mediate $\mathrm{GABA}_{\mathrm{A}, \text { slow }}$ currents on type $P$ cells.

In this study, in contrary to [20], type I' population is confined to oriented dendrite-targeting cells mediating $\mathrm{GABA}_{\mathrm{A} \text {,slow }}$ currents on pyramidal cells. The $\mathrm{GABA}_{\mathrm{B}}$ receptor, which is mostly provided by neurogliaform cells, was not included in type I' population since we were interested in the very early peaks of the EP responses.

A schematic of the model including the three above-mentioned neuronal sub-populations and their interconnections is provided in Fig. 1. Interactions between the three sub-populations are characterized by connectivity constants $C_{P P}, C_{P I}, C_{I P}, C_{I I}, C_{P I^{\prime}}, C_{I^{\prime} P}, C_{I^{\prime} I^{\prime}}$ and $C_{I^{\prime} I}$ which account for the average number of synaptic contacts between sub-populations.

In the model, the sub-cortical input is represented by a quantity which describes the density of action potentials coming from sub-cortical structures to the SSC. This quantity is assumed to abruptly increase due to air-puff stimulation. In response to the sub-cortical input, both type I and $I$ ' interneurons provide feed-forward inhibition to pyramidal cells in addition to the classical feed-back inhibition. Both feed-forward and feed-back inhibitory processes were found to be present in the somatosensory (S1) "barrel" cortex of rodents and rabbits [21]. Feed-forward inhibition is the fastest one. It is generated by monosynaptic thalamic input driving cortical inhibitory interneurons (in addition to targeted pyramidal cells in S1).

A summation of average postsynaptic potentials at the level of pyramidal cells $\left(v_{P}\right)$ is considered as the model output. Indeed, it can be assumed [25] that the temporal dynamics of this simulated signal corresponds to that of the actual LFP (quasi-static assumption) which is the essential point in the problem we tackle (dynamics of the EP, i.e. relative amplitudes and 
latencies of the main peaks). Readers may refer to the appendix for a more detailed description of the model as well as for the mathematical equations.

\subsubsection{Representation of the effect of tDCS in the model}

In addition to the effect of a sensory input (i.e., induced by the air-puff), the effect of the applied tDCS electric field on the cerebral cortex was also included in the proposed model. The influence of tDCS was represented as a perturbation on the mean membrane potential of neuronal sub-populations included in the model (pyramidal cells and interneurons). It was also assumed that, within a certain range of magnitude, the applied electric field modifies the mean membrane potential of sub-populations in a linear way. In addition, this modification is direction-dependent [8]. More specifically as shown in Fig. 2, a field aligned with the orthodromic direction (dendritic tuft to axon), will result in a positive (depolarizing or excitatory) perturbation of the soma membrane potential. Conversely, a field in the antidromic direction will have a negative influence (hyperpolarizing or inhibitory).

According to the above assumptions, it can be written that $\Delta V \approx \vec{\lambda} \cdot \vec{E}$, for some $\lambda$ representing the "effective" membrane space constant and pointing in the "orthodromic" direction of the neuron. This model, often referred to as the " $\lambda E$ model", is well grounded in the biophysics of compartment models [9-12] (see [6] for a review) and supported by in-vitro experiments $[8,26]$. In our model, the above considerations led us to consider the perturbation of the mean membrane potential of pyramidal cells as a linear function of the externally-applied field magnitude, although our in-vivo setup could not allow us to experimentally measure $E$ and $\lambda$ as well as their spatial distribution. The same linear effect was also considered on interneuron populations which were shown to also have a "preferred" orientation [27-30]. Formally, we added the term $v_{X}^{t D C S}$ to the membrane potential $v_{X}$ where $X \in\left\{P, I, I^{\prime}\right\}$ (tDCS may affect the 
subpopulations differently). This differential voltage term (i.e., $\Delta v_{X}=v_{X}^{t D C S}$ ) may have either a depolarizing or a hyperpolarizing effect on a given sub-population, depending on its polarity. As a consequence, the firing rate of neuronal populations described by the wave-to-pulse sigmoid function directly increases or decreases accordingly. It is worth mentioning that this effect on the firing rate is also consistent with results reported experimentally [8], as the applied field also modifies the action potential threshold.

\section{Results}

\subsection{Experimental data: evoked responses and effects of tDCS}

Fig. 3A-C (left) displays some typical single-trial local field potential responses under control (no tDCS stimulation), anodal tDCS, and cathodal tDCS conditions. The averaged somatosensory evoked potential (aSEP) for these conditions $(n=10)$ is shown on the right. A reduced number of epochs was chosen in order to focus on immediate effects of the externally-applied field and to avoid the corruption of SEPs by long-term effects of tDCS currents on brain activities [31]. Fig. 3D provides a superposition of aSEPs in control condition as well as in anodal and cathodal tDCS conditions. The interval between $15 \%$ to $85 \%$ percentiles of SEP values for each tDCS condition is shown by a color-coded patch: green for the control condition, light red for the anodal current, and dark blue for the cathodal current. Under control condition, the aSEP was characterized by a high-amplitude negative potential $(N 1)$ that peaked at about $4 \mathrm{~ms}(N 1 a)$ and $14 \mathrm{~ms}(N 1 b)$ followed by three loweramplitude positive and negative peaks at different latencies $(P 1: 32 \mathrm{~ms}, N 2: 56 \mathrm{~ms}, P 2: 82$ ms). Under tDCS, the features of the aSEP were modified. The amplitude of the first prominent negative peak changed about $20 \%$ under anodal and cathodal tDCS currents compared to that recorded under control condition. Anodal current induced an increase in the amplitude of N1, whilst cathodal current induced an opposite effect on N1 (in control, anodal 
and cathodal conditions the amplitude of $N 1 a$ was $0.93 \pm 0.28,1.18 \pm 0.21,0.78 \pm 0.29 \mathrm{mV}$ and the amplitude of $N 1 b$ was $0.89 \pm 0.2,1.09 \pm 0.19$ and $0.76 \pm 0.2 \mathrm{mV}$, respectively. tDCS also affected late small-amplitude components of the aSEP (i.e., P1-N2-P2 complex). The first positive peak in control condition $(P l)$ diminished a little bit when a tDCS current (either anodal or cathodal) was applied on the brain (control: $0.55 \pm 0.11 \mathrm{mV}$, anodal: $0.41 \pm 0.23 \mathrm{mV}$, cathodal: $0.42 \pm 0.13 \mathrm{mV}$ ). Anodal stimulation slightly increased the frequency of the P1-N2$P 2$ complex (the intervals between $P 1$ and $P 2$ peaks in control and anodal conditions were 50 and $40 \mathrm{~ms}$, respectively), whilst cathodal current mainly decreased this frequency up to a point where a merging of $P 1, N 2$ and $P 2$ into a single positive peak $P$ could be observed.

\subsection{Simulation of the evoked potential under "no tDCS stimulation" conditions}

As a first step, the computational model was explored in order to determine a first set of parameters for which the actual aSEP could be approximated. The optimization procedure was first performed manually based on the visual analysis of the simulated EP (Fig. 4A) as well as the average EPSP and IPSP components (slow and fast, Fig. 4B) of this EP, at the level of pyramidal cells. This qualitative optimization procedure was then complemented by a parameter sensitivity analysis aimed at studying the impact, on the simulated EP, of random changes affecting the parameter vector $\boldsymbol{\theta}=\left\{A, B, G, n_{P}, n_{I}, n_{I}\right\}$ (see the appendix for parameter definition). As shown in Fig. 4C, results show that the simulated EP response stays "quite robust" (in the sense that the general shape is conserved) when parameters stay in the range of $\left[\boldsymbol{\theta}_{0} \pm \alpha . \boldsymbol{\theta}_{0}\right]$ with $\alpha<0.5$.

Table 1 shows the numerical values of the model parameters corresponding to the EP in Fig. 4. Specific sets of parameter values corresponding to similar mechanisms in the model could be identified leading to the simulation of an EP which closely approximated the actual aSEP 
(in terms of peaks and associated latencies). Indeed, i) very specific EPSP, fast IPSP and slow IPSP time-courses must be summed up in order to produce a simulated EP with correct peak polarities at right latencies, as shown in Fig. 4 and ii) the number of parameter combinations leading to these three specific PSP waveforms was found to be low and could be identified without the need to use sophisticated identification procedures. In fact, the detailed analysis of the simulated EP and its EPSP and IPSP components revealed that the first major negative peak (N1) was basically generated when the mean firing rate of type $P$ population started to increase in response to the excitatory sub-cortical input (air-puff effect) that targets the pyramidal cell population (type $P$ ). A transient biphasic decrease and increase of excitatory input at the level of type $P$ population generates Nla and N1b sub-peaks. This transient effect is mostly related to the inhibitory path that connects type $I^{\prime}$ interneurons to type $I$. The difference between decay-times of $\mathrm{GABA}_{\mathrm{A} \text {,fast }}$ and AMPA synapses can also reinforce this effect (inhibitory and excitatory inputs appear with different decay rates at the level of type $P$ population).

Next, the appearance of the first positive peak $P 1$ in the EP was due to a late secondary increase of inhibitory signals on pyramidal cells. This inhibition was basically initiated by type I' population, but type I population reinforced this late inhibitory effect on type $P$ population. Indeed, at this stage type I population has recovered from the inhibited state that had been induced by type I' before. This recovery was basically provided by the interconnections within type I interneurons. Finally, the return to baseline and the generation of small $N 2$ and $P 2$ peaks were due to interplay between pyramidal cells and interneurons subpopulations and changes in the net value of incoming firing rate at the level of type $P$ population. This change was provided by differences between the decay rates of EPSP, fast IPSP and slow IPSP components. 


\subsection{Simulation of tDCS effects on simulated evoked potentials}

In this section, the objective was twofold: i) to reproduce, in the model, the main effects of tDCS as observed in real data and, subsequently ii) to get some insight into the impact of externally-applied fields on neuronal populations based on the analysis of model parameters for which "realistic" effects could be reproduced.

As described earlier in section 2.2.2, tDCS short-time effects stem from the stimulation acting as a source of perturbation on the mean membrane potential of neuronal sub-populations. Our approach was progressive: this perturbation was first introduced at the level of the pyramidal cell population only, hypothesizing that the influence of externally-applied fields was mainly on these pyramidal cells due to their preferred orientation in the cortex (section 3.3.1). Then, it was assumed that this perturbation could also affect the two types of interneuron subpopulations (section 3.3.2).

\subsubsection{Effect of tDCS on the EP: Only pyramidal cells sub-population is impacted (situation 1)}

Starting from the set of model parameters for which the EP under no tDCS stimulation could be reproduced to a good extent, it could be qualitatively observed that both the latencies and the peak amplitudes of the simulated EP varied when a positive or negative DC-offset (mimicking the effect of anodal or cathodal tDCS) was applied on the mean membrane potential of the pyramidal cell subpopulation.

The setting of model parameters was found to affect the characteristics of these variations. In particular, the balance between the respective contribution of the positive and the two negative loops in the model was found to be a determining factor in the EP waveform under tDCS conditions. Starting from this observation, we investigated which configurations of model parameters obeyed the two following constraints: i) generate a realistic EP waveform 
under the control condition, and ii) reproduce some of the features of actual aSEP waveforms under tDCS (realistic modifications of peak amplitudes and latencies), as described in section 3.1 and summarized in table 2 (first column).

Empirically, four classes of model configurations (and hence of simulated EP responses) could be identified which fulfilled the two above constraints, but only partially. Fig. 5 illustrates a representative EP response for each of these four classes under control, cathodal and anodal tDCS stimulation. Each class is briefly described below along with its ability to reproduce the features (peak amplitudes and latencies, changes w.r.t. anodal/cathodal stimulation) observed in actual aSEPs.

The first class of EP responses (Fig. 5A) was observed when the contribution of the positive feedback in the $P-P$ loop (where $P$ stands for pyramidal cells) was high enough, and the inhibitory effect of type $I$ ' to type $P$ population was very strong. In this class and in other three subsequent classes of EP responses that are described hereafter, the amplitude of Nla was not prominently modified by tDCS current mostly because early depolarization of type $P$ population in response to air-puff effect is not much affected by tDCS. In the first class of EP responses, the amplitude of $N 1 b$ was increased by the anodal tDCS current. Indeed the strong positive feedback which increased with the anodal tDCS field (because $P$ cells are more depolarized) was the main factor for the increase of the amplitude of $N 1 b$ peak. On the other hand, the strong connection between type I' and type $P$ caused an increase of the amplitude of $P 1$ peak in the anodal tDCS condition.

In the second class of EP responses (Fig. 5B), the effective gain of the positive $P-P$ feedback was reduced, for example, by a reduction in the value of the $C_{P P}$ parameter. Reducing this gain increased the contribution of negative feedbacks and facilitated the interplay between pyramidal cells and interneurons to generate prominent $P 1, N 2$ and $P 2$ peaks in the EP. In 
addition, this modification resulted in the increase of the frequency of $P 1-N 2-P 2$ complex under anodal tDCS stimulation (this effect could not be reproduced in the first class). On the other hand, the increase of the amplitude of $N 1 b$ peak in the anodal tDCS stimulation was less important because the gain of positive $P-P$ feedback was reduced. As in the first class, the amplitude of $P 1$ peak increased after application of anodal current, contrarily to the actual effect observed in the rabbit (table 2).

The third class corresponded to the situation in which the amplitude of $P 1$ peak decreased under anodal tDCS, as observed in real signals. To fulfill this constraint, the gain of positive feedback had to be high enough, and interneurons (especially type I') should not strongly inhibit type $P$ subpopulation. However, this situation increased the amplitude of $P 1$ peak and reduced its latency, which was not in agreement with real aSEP data. Since in the third class of EP responses the contribution of positive feedback is high, the amplitude of $N 1 b$ prominently increased with the anodal tDCS current. The frequency of $P 1-N 2-P 2$ complex increased with anodal tDCS. Both of these simulated effects matched the effects observed in real aSEP data.

Finally, for the fourth class of EP responses, the structure of the model was slightly modified compared to the third class, so that the sensitivity of type I' population to respond to an incoming excitatory input was increased (for example, by increasing the value of $\theta_{I^{\prime}}$ and $r_{I^{\prime}}$ ). The characteristics of EP responses in this fourth class were very similar to those in the third class. The main difference was in the latency of the $P 1$ peak. Indeed, in the third class the latency of $P 1$ peak under anodal tDCS was greater than that under cathodal tDCS, whereas, in the fourth class, this latency increased for the cathodal tDCS. In this respect, EP responses belonging to the fourth class were more similar to real aSEP responses. However, it should be 
mentioned that even by reducing the sensitivity of type I' interneurons, the increase in the $P I$ peak latency was not enough to reach the $P 1$ latency in real data.

The above results, summarized in table 2 , showed that the model could hardly reproduce the actual EP (obtained under control and under the anodal/cathodal stimulation conditions) when the perturbation of the mean membrane potential was applied only on pyramidal cells. These considerations led us to study whether the three major discrepancies could be enhanced or even suppressed when the externally-applied field resulting from tDCS influenced both pyramidal and inhibitory interneuron subpopulations.

\subsubsection{Effect of tDCS on the EP: All sub-populations are considered to be impacted (situation 2)}

From situation 1 (see table 2), it could be inferred that i) the increase (resp. decrease) of Nla amplitude was difficult to reproduce under anodal (resp. cathodal) stimulation, ii) the decrease of $P 1$ amplitude was not seen in both conditions and iii) the augmentation of the latency of $P 1$ under cathodal stimulation was not reproduced by the model. These three limitations were used as extra-constraints in an optimization procedure aiming to determine whether the agreement between simulated EPs and real aSEPs could be improved when the mean membrane of all subpopulations in the model were subjected to perturbation by tDCS current. According to this quantitative procedure, we plotted the evolution of EP features when the 2D parameter space corresponding to the change of mean membrane potential of type I and type I' interneurons was covered, under each stimulation condition. The resulting 3D plots (Fig. 6 and Fig. 7) reveal how these two parameters should be changed to correct the aforementioned discrepancies (i-iii) while keeping the other features in agreement with real ones.

Fig. 6 illustrates the variation of $N 1 a, N 1 b, P 1$ and $N 2$ amplitudes induced by anodal (left panels) and by cathodal (right panels) tDCS. A positive (resp. negative) value in any panel in 
this figure indicates an increase (resp. decrease) in the absolute amplitude value of a given peak compared to that in the control condition. In a similar manner, the variation of Nla, N1b, $P 1$ and N2 latencies under anodal and cathodal tDCS is illustrated in Fig. 7. The type P population was assumed to be depolarized (resp. hyperpolarized) by the anodal (resp. cathodal) current (section 2.2.2). Conversely, in absence of precise knowledge about the field effects on interneurons, it was assumed that both type $I$ and type $I^{\prime}$ subpopulations could be subjected to both depolarization and hyperpolarization with different strengths regardless the polarity of tDCS stimulation.

Regarding N1 peak, results reported in Fig. 6 and Fig. 7 indicated that changes in the mean membrane potential of type I and type I' interneurons did not drastically change the latency of $N 1 a$ and N1b peaks, but could modify the amplitude of these peaks. Modification of the amplitude of $N 1 a$ was more sensitive to the polarization of type $I$ than type $I^{\prime}$ subpopulation. This effect could be explained by an amplification of the fast feed-forward inhibitory process (input to type $P$ subpopulation by type $I$ subpopulation) by depolarization of type $I$ interneurons leading to higher-amplitude fast IPSPs on pyramidal cells and subsequently to prominent change in the LFP (early Nla peak). Conversely, the hyperpolarization of type I subpopulation increased the amplitude of N1a peak (Fig. 6A right). Indeed, hyperpolarized type I interneurons mediated weaker fast feed-forward IPSPs on type $P$ subpopulation. Hence, the effect of excitatory sub-cortical inputs on type $P$ increased and led to enlarge the amplitude of Nla. This results suggests that if type I population is hyper-polarized (resp. depolarized) in anodal (resp. cathodal) tDCS stimulation, EP simulated in the computational model were more consistent with real EPs.

General inspection of Fig. $6 \mathrm{~B}$ indicated that $N 1 b$ peak was more sensitive to the polarization of type $I^{\prime}$ interneurons than to the polarization of type $I$ interneurons. Moreover, Fig. 6B (left) 
revealed that the mean membrane potential of type I' subpopulation should be augmented in order to get an increase of the amplitude of N1b peak under anodal stimulation, as in the real case. Along the same lines, Fig. 6B (right) showed an opposite effect on N1b when the type I' subpopulation was hyperpolarized by cathodal stimulation. This result suggests that tDCSinduced changes in the second early peak of the simulated EP better matched the modifications induced by tDCS in the real aSEP when appropriate changes were applied on the mean membrane potentials of type I' interneuron subpopulation.

Regarding $P 1$ peak, its amplitude increased by anodal tDCS stimulation if both type $I$ and type I'subpopulations were depolarized (Fig. 6C left). Conversely, hyperpolarization of these two subpopulations by cathodal tDCS current decreased the amplitude of $P 1$ peak (Fig. 6C right). Since a maintaining (or even the reduction) of $P 1$ amplitude was observed in the real aSEP for both anodal and cathodal tDCS, we could deduce from Fig. 6C that polarizations of type $I$ and type $I^{\prime}$ subpopulations should be in opposite directions.

Finally, regarding the latency of $P 1$ peak, Fig. $7 \mathrm{C}$ showed that polarization of type $I^{\prime}$ interneurons was more important than the polarization of type $I$ interneurons, as this peak is basically initiated and controlled by the activity of type $I^{\prime}$ interneurons. As this latency should increase to better match the observed effect during cathodal tDCS, it could be inferred from the plot in Fig. 7C (right) that type $I^{\prime}$ subpopulation should be hyperpolarized. In the model, this effect could be explained as follows. When type $I^{\prime}$ subpopulation was hyperpolarized by cathodal tDCS, it became "less sensitive" to incoming excitatory signals (either from feedforward or pyramidal cell input) and thus reduced its firing rate. As a consequence, the decrease of IPSPs on type $P$ subpopulation resulted, at the level of the EP, in a slight decrease of $P 1$ amplitude and increase in $P 1$ latency. Anodal tDCS was found to have an opposite effect on the latency of $P 1$ peak. 


\subsection{Optimal simulation of tDCS effects}

In order to quantitatively determine how should interneuron populations be polarized such that simulated EPs under tDCS still match actual EPs, we designed an optimization procedure. Based on a cost function, this procedure minimizes the difference between simulated and real amplitudes / latencies of $N 1 a, N 1 b, P 1$, and $N 2$ peaks wheb the three neuronal sub-populations get polarized. Fig. 8 shows the results (as 2D maps) obtained for full polarization coverage of mean membrane potential of type $I$ and type $I$ ' interneurons under anodal and cathodal tDCS. The best performance was observed when both types of interneurons in the model were polarized with tDCS, the polarization of type $I^{\prime}$ population being more important than that of type $I$. Under anodal tDCS conditions, type $P$ and type $I^{\prime}$ populations should be depolarized, and hyperpolarization of type $I$ enhanced the results. Under cathodal tDCS conditions, these polarizations should be inversed.

Finally, Fig. 9 illustrates three EPs simulated under cathodal, control and anodal tDCS currents when all the three subpopulations were influenced according to the "optimal" polarization conditions. The same parameter values given in table 1 were used to simulate these EP responses. The net polarization effect of tDCS on the type $P$ subpopulation was $+4 \mathrm{mV}$ and $-4 \mathrm{mV}$ during anodal and cathodal tDCS. This amount of polarization corresponds to the polarizing effect of an electric field equal to $30 \mathrm{mV} / \mathrm{mm}$ in the in-vivo experiments [8]. The polarization effect of tDCS on type I and type I' subpopulation was $35 \%$ and $50 \%$ of the polarization effect of tDCS on type $P$ subpopulation, respectively (i.e., $v_{P}^{t D C S}= \pm 4 m V$, $\left.v_{I}^{t D C S}=\mp 1.4 m V, v_{I^{\prime}}^{t D C S}= \pm 2 m V\right)$.

\section{Discussion and conclusion}


Elucidating the processes by which weak exogenous electric fields (induced by tCS) affect ongoing brain activity is a key issue for the rational design of stimulation devices and strategies [32]. A number of recently-published experimental studies [33, 34] have confirmed that tCS modifies the excitability of neurons (through changes of membrane potential and firing rate) and hence the resulting cortical activity. However, it is well admitted that physiological mechanisms of how tCS affects and interacts with the activity of neuronal populations remain elusive. At the level of single pyramidal neurons, based on in-vitro work, some effects of electric fields (DC and AC) could be relatively well described in terms of orientation and polarity, changes in trans-membrane potential, modification of firing rates, site of action potential initiation [35]. However, at the level of neuronal networks, precise information is still missing. More particularly, a question still unsolved is how the applied electric fields affect the behavior of non-pyramidal neurons (typically inhibitory interneurons) that modulate the activity of pyramidal cells which are themselves known to be strongly influenced by the fields [36].

In order to address this issue, we have used a computational modeling approach combined with experimental data.

Given the proposed model architecture, the main findings, discussed below, can be summarized as follows: (i) early responses observed in the EP recorded in the SSC result from the activation of a network of pyramidal cells and interneurons (2 main types) interacting via glutamatergic (AMPA) and GABAergic (GABAa receptors) processes, (ii) tDCS does not only affect pyramidal cells, but also interneurons, (iii) polarization of type $I^{\prime}$ is necessary to reproduce EPs with correct features under tDCS, and, (iv) if type I interneurons are polarized in the opposite way, the optimum result is obtained. 
Regarding our stimulation/recording experimental protocol, the objective was to measure local electrical activities of a small brain area under tDCS-like conditions. It is worth mention that in clinical applications tDCS is usually applied by means of a large surface electrode to the scalp. Such a configuration could not be strictly reproduced in our rabbit experiment due to several technical limitations. Firstly, the skin of the rabbit is not as well fixed to the skull as in humans, therefore, electrodes were placed directly above the bone in order to ensure a fixed localization; Moreover, our protocol has been designed to simultaneously (i) stimulate a brain region with tDCS-like currents and (ii) record local electrical activities arising from this small brain area. As a result, we applied DC currents through 4 silver electrodes placed above the skull around the drilled hole. Such a configuration induces a focused stimulation of the somatosensory region, whereas in clinical tDCS studies, the use of a large surface electrode would result in stimulating a larger brain area. Despite these methodological discrepancies, and although there is no result concerning the immediate effects of tDCS on human somatosensory cortex (to the best of our knowledge), longer-term effects reported in clinical studies can be related with the effects we observed in rabbits. In particular, a reduction (resp. enlargement) of sensory evoked potential components have been previously described after cathodal (resp. anodal) tDCS $[37,38]$.

Regarding the modeling approach, we developed a physiologically-plausible macroscopic (i.e., neuronal population) model based on a previously designed model [20], but including a fast feed-forward inhibitory mechanism. Secondly, we used this model to reproduce real aSEP recorded in rabbits, and to analyze underlying cortical mechanisms at the origin of observed EPs at the level of interactions between sub-populations of neurons that constitute the neuronal population from which the LFP is recorded. Finally, this model accounted for the effects of tDCS on neuronal subpopulations. To the best of our knowledge, these three aspects are original. Although population models have considerably been used to study different brain 
rhythms in normal and clinical conditions such as in waking and resting state [39], sleep [40], anesthesia [41, 42], epilepsy [43, 44], and even in event related responses [45, 46], they have not been utilized commonly for exploring the interaction between externally applied fields and neuronal activities. A first attempt was reported in [47] in the context of seizure anticipation based on an active paradigm that uses intermittent direct electrical stimulation.

One of the interesting points we faced while developing the model was the necessity of feedforward inhibition for generating the two early narrow negative peaks observed in the EP $(N 1 a$ and $N 1 b)$. This result corroborates the fact that this type of inhibition has been reported to actually exist in the SSC of several species $[22,48]$. We also noticed that if the inhibitory population which provides the fast feed-forward inhibition on pyramidal cells (i.e., type I interneurons) is not inhibited by the other inhibitory population, it would be difficult to generate $N 1 a$ and $N 1 b$ peaks with the narrow valley between them. Again, this type of synaptic interaction in the cortex, for instance, from bipolar toward basket cells locating in infragranular layers of SSC has been identified [49]. These observations highlight the fact that brain stimulation can provide a very valuable tool to study such coupling mechanisms.

We investigated the possible effects of anodal and cathodal tDCS on neuronal subpopulations, the objective being to minimize the distance between features (peaks, polarity, latencies) of simulated and real EPs. We noticed that there exists a relation between the best configuration according to which we perturb membrane potentials of neuronal populations to get the "optimum" results in the model, on the one hand, and the geometrical properties of these neuronal populations in the brain, on the other hand. Indeed, we could observe, in the model, that pyramidal cells constitute the neuronal sub-population that is affected the most by tDCS.

In the model, pyramidal cells should be depolarized so that we can simulate the effect of anodal currents on EPs and they should be hyperpolarized so that we can simulate the effect 
of cathodal currents. These findings are consistent with biophysical arguments. Any current that is injected from scalp electrodes will produce an electric field component along the somato-dendritic axis of pyramidal cells. Depending on the orientation of this field component, it can be shown that the resulting effect is either a de- or a hyper-polarization of the cells.

Interestingly, in the model, results also revealed that the externally-applied field is also likely to affect interneurons. This is an important - still unsolved - issue that is often addressed in studies related to the effects of weak fields on the brain. We found out that if interneurons are polarized then the characteristics of simulated EPs become closer to those of real EPs, indicating that modeling efforts need to consider the "less understood" role of these neuron types. Geometrical characteristics of interneurons may provide some explanations for this. Indeed, some types of interneurons such as large basket cells, bitufted cells and bi-polar cells have oriented dendritic trees $[27,50]$. Some bitufted cells as shown in [51] have a dense dendritic structure with aligned branches in one side of the soma, and large basket cells as shown in $[27,50]$ usually have some parallel long dendrites directing toward white matter. This oriented structure of dendritic targeting slow-interneurons and somatic targeting fastinterneurons can explain why a better result is obtained in the model when type $I$ and type I' inhibitory interneurons are polarized under tDCS conditions. Regarding the experimental verification of model predictions, a possible in-vivo experiment aimed at modulating the activity of the SSC based on local injection of drugs can be performed. This would specifically affect the synaptic transmission, and subsequently the time-course of actual EPs which could be compared to simulated ones obtained for alteration of EPSP and IPSP parameters. Besides, experiments assessing the specific effects of tDCS on interneurons of different types and on neuronal networks are rather limited. One of the most relevant experiment w.r.t results obtained from our modeling study is that conducted by [36] which 
also suggests that interneurons are also affected by tDCS and that opposite polarization may occur for the same tDCS polarity.

We focused on short-term (immediate) effects of tDCS on EPs. Therefore, we only analyzed the EPs that were recorded during the first minutes just after the application of anodal or cathodal currents. The study of long-term effects is beyond the scope of this paper but can be performed with the proposed model as a detailed analysis of potentiation- and/or depressionrelated parameters (for instance the amplitude of average post-synaptic potentials) using both computational and experimental approaches. Finally, since our model only accounts the temporal (and not spatial) properties of the brain activity, polysynaptic effects (possibly occurring when multiple sources are activated) are not considered. A possible way to extend the model in this aspect is to implant electrodes in more than one region (e.g., in S2) to obtain a spatial pattern of LFP and tDCS current. Nevertheless, it is unlikely that polysynaptic effects can play a major role in the formation of high-amplitude short-latency peaks in the EP. For later responses, which are not in the main focus of this study, polysynaptic may have some effects.

\section{Acknowledgements}

The project HIVE acknowledges the financial support of the Future and Emerging Technologies (FET) program within the Seventh Framework Program for Research of the European Commission, under FET-Open grant number: 222079 (http://hive-eu.org/). 


\section{Appendix}

In the model, the input of each sub-population is described by bi-exponential pulse-to-wave functions (mimicking the average excitatory and inhibitory post-synaptic potentials - PSPs -). Indeed, each of these functions describes how a pre-synaptic pulse density of afferent action potentials $Q_{c, X}(t)$ is transformed into a post-synaptic membrane potential $\varphi_{c, Y}(t)$ inside the cortex $c$ (see Fig. 1B for pyramidal cells population). The following four equations describe the relations between $Q_{c, X}(t)$ and $\varphi_{c, Y}(t)$. They depend on the topology of the model (i.e. the way the three sub-populations are synaptically interconnected). $X \in\left\{P, I, I^{\prime}\right\}$ denotes the considered sub-population type (type $P$, type $I$ or type $I^{\prime}$ ) and $Y \in\left\{P_{a, \tilde{a}}, I_{g, \tilde{g}}, I_{g, \tilde{g}}^{\prime}, I_{b, \tilde{b}}^{\prime}\right\}$ refers to the type of synaptic interaction (including the name of the source sub-population, the neurotransmitter type and associated receptor kinetics $\{a, \tilde{a}\}:$ AMPA, $\{g, \tilde{g}\}: \mathrm{GABA}_{\mathrm{A}, \text { fast }}$ and $\left.\{b, \tilde{b}\}: \mathrm{GABA}_{\mathrm{A}, \mathrm{slow}}\right)$.

$\left(\frac{\partial^{2}}{\partial t^{2}}+(a+\tilde{a}) \frac{\partial}{\partial t}+a \tilde{a}\right) \varphi_{c, P_{a, \tilde{a}}}(t)=A \tilde{a} c_{(a, \tilde{a})} Q_{c, P}(t)$

$\left(\frac{\partial^{2}}{\partial t^{2}}+(g+\tilde{g}) \frac{\partial}{\partial t}+g \tilde{g}\right) \varphi_{c, I, \tilde{g}, \tilde{g}}(t)=G \tilde{g} c_{(g, \tilde{g})} Q_{c, I}(t)$

$\left(\frac{\partial^{2}}{\partial t^{2}}+(g+\tilde{g}) \frac{\partial}{\partial t}+g \tilde{g}\right) \varphi_{c, I_{g, \tilde{g}}^{\prime}}(t)=G \tilde{g} c_{(g, \tilde{g})} Q_{c, I^{\prime}}(t)$

$\left(\frac{\partial^{2}}{\partial t^{2}}+(b+\tilde{b}) \frac{\partial}{\partial t}+b \tilde{b}\right) \varphi_{c, I_{b, \tilde{b}}^{\prime}}(t)=B \tilde{b} c_{(b, \tilde{b})} Q_{c, I^{\prime}}(t)$

The generic form of the bi-exponential functions is as follows:

$h(t)= \begin{cases}c_{(w, \tilde{w})} \tilde{w} W \frac{e^{-w t}-e^{-\tilde{w} t}}{\tilde{w}-w} \Theta(t) & \text { if } w \neq \tilde{w} \\ c_{(w, \tilde{w})} w W t e^{-w t} \Theta(t) & \text { otherwise }\end{cases}$ 
In the above equation, $w$ can be one of the $a, b$ and $g$ parameters, $W$ may be one of the $A$, $G$ and $B$ parameters, and $\Theta(t)$ denotes the Heaviside function. $c_{(w, \tilde{w})}$ is a normalizing constant value which is given by the following equation:

$c_{(w, \tilde{w})}= \begin{cases}\left(\frac{\tilde{w}}{w}\right)^{\frac{w}{\tilde{w}-w}} & \text { if } w \neq \tilde{w} \\ \exp (1) & \text { otherwise }\end{cases}$

This normalizing factor sets the maximum value of the bi-exponential function $h(t)$ to $W$. In the PSP functions used in this model were represented by bi-exponential functions in which the rise and decay times of the can be set independently [23]:

$t_{\text {rise }}=E /(\tilde{w}-w)$

$t_{\text {decay }}=t_{\text {rise }}\left(0.90211+2 \frac{\sinh (E)}{E}+0.30538 \frac{\tanh \left(0.7992^{*} E\right)}{E}\right)$

where $E=\ln (\tilde{w} / w)$, and $t_{\text {rise }}$ is the time at which PSP takes its maximum value $W$, and $t_{\text {decay }}$ is the time at which it goes down to $37 \%$ of $W$.

In the model, sub-cortical input is accounted for by the quantity $Q_{s, X}(t)$ (where $X \in\left\{P, I, I^{\prime}\right\}$ ) which represents the density of action potentials coming from sub-cortical structures $s$ to the three types of sub-populations and which abruptly increases in the presence of the air-puff stimulation. Mathematically $Q_{s, X}(t)$ is expressed as below:

$Q_{s, X}(t)=m_{X}+n_{X} \underbrace{e^{-t / \tau} \Theta(t)}_{p(t) \text { air-puff effect }}$ 
where $m_{X}$ controls the average background firing rate and where $n_{X}$ controls the gain of airpuff effect. The induced post-synaptic potential $\varphi_{s, X}$ by the driving sub-cortical input signal $Q_{s, X}(t)$ is given as follows:

$\left(\frac{\partial^{2}}{\partial t^{2}}+(a+\tilde{a}) \frac{\partial}{\partial t}+a \tilde{a}\right) \varphi_{s, X}(t)=A \tilde{a} c_{(a, \tilde{a})} Q_{s, X}(t)$

The mean membrane potential of each sub-population is calculated by one of the following three equations. These equations describe the influence of post-synaptic potentials $\varphi_{c, Y}(t)$ and $\varphi_{s, X}(t)$ resulting from both intra-cortical and sub-cortical interactions, respectively, on the mean membrane potentials $v_{P}$ (model output), $v_{I}$ and $v_{I^{\prime}}$ of the three sub-populations $P, I$ and $I^{\prime}$.

$$
\begin{aligned}
& v_{P}=\varphi_{c, P_{a, \tilde{a}}} C_{P P}+\varphi_{c, I_{g, \tilde{s}}} C_{I P}+\varphi_{c, I_{b, \tilde{b}}^{\prime}} C_{I^{\prime} P}+\varphi_{s, P} \\
& v_{I}=\varphi_{c, P_{a, \tilde{a}}} C_{P I}+\varphi_{c, I_{g, \tilde{g}}} C_{I I}+\varphi_{c, I_{g, \tilde{g}}^{\prime}} C_{I^{\prime} I}+\varphi_{s, I} \\
& v_{I^{\prime}}=\varphi_{c, P_{a, \tilde{a}}} C_{P I^{\prime}}+\varphi_{c, I_{b, \tilde{b}}^{\prime}} C_{I^{\prime} I^{\prime}}+\varphi_{s, I^{\prime}}
\end{aligned}
$$

At the output of each sub-population a wave-to-pulse function transforms the mean membrane potential of the soma to a firing rate quantity. In general, the firing rate monotonically increases with mean soma membrane potential. Based on this general property of neuronal cells, the wave-to-pulse function is usually represented by a static nonlinear increasing function of sigmoid shape $[18,24]$ to represent threshold and saturation effects taking place at the soma. The generic form of this function is as follows:

$Q_{X}\left(v_{X}\right)=Q_{X}^{\max } /\left(1+e^{r_{X}\left(\theta_{X}-v_{X}\right)}\right)$ 
$X$ refers to one of the $P, I$ and $I^{\prime}$ sub-populations. $Q_{X}^{\max }, \theta_{X}$ and $r_{X}$ represent maximum firing rate, inflection point of the sigmoid function, and the slope at the inflection point, respectively.

In practice, the model consists of 14 connected first-order ordinary differential equations with nonlinear terms introduced by the sigmoid functions. These equations were solved using a fourth-order Runge-Kutta algorithm (fixed step size). 


\section{References}

[1] A. Rogalewski, C. Breitenstein, M. A. Nitsche, W. Paulus, and S. Knecht, "Transcranial direct current stimulation disrupts tactile perception," Eur J Neurosci, vol. 20, pp. 3136, 2004.

[2] M. A. Nitsche, L. G. Cohen, E. M. Wassermann, A. Priori, N. Lang, A. Antal, W. Paulus, F. Hummel, P. S. Boggio, F. Fregni, and A. Pascual-Leone, "Transcranial direct current stimulation: State of the art 2008," Brain Stimul, vol. 1, pp. 206-23, 2008.

[3] G. Csifcsak, A. Antal, F. Hillers, M. Levold, C. G. Bachmann, S. Happe, M. A. Nitsche, J. Ellrich, and W. Paulus, "Modulatory effects of transcranial direct current stimulation on laser-evoked potentials," Pain Med, vol. 10, pp. 122-32, 2009.

[4] L. Marshall, M. Molle, M. Hallschmid, and J. Born, "Transcranial direct current stimulation during sleep improves declarative memory," J Neurosci, vol. 24, pp. 998592, 2004.

[5] A. Riera, J. Llobera, I. Cester, D. Whitmer, M. Bracewell, N. Davis, E. Angelakis, E. Liouta, T. Penzel, and C. Schoebel, Human brain stimulation: Technologies and clinical applications, Deliverable D3.1: HIVE (FET Open FP7 EU project), Available at http://hive-eu.org, 2009.

[6] P. C. Miranda, F. Wendling, G. Ruffini, I. Merlet, B. Molaee-Ardekani, S. Dunne, A. Soria-Frisch, and D. Whitmer, Brain stimulation: models, experiments and open questions, Deliverable D1.1: review of the state of the art in currents distribution and effects HIVE (FET Open FP7 EU project), Available at http://hive-eu.org, 2009.

[7] L. J. Bindman, O. C. Lippold, and J. W. Redfearn, "The Action of Brief Polarizing Currents on the Cerebral Cortex of the Rat (1) During Current Flow and (2) in the Production of Long-Lasting after-Effects," J Physiol, vol. 172, pp. 369-82, 1964.

[8] M. Bikson, M. Inoue, H. Akiyama, J. K. Deans, J. E. Fox, H. Miyakawa, and J. G. Jefferys, "Effects of uniform extracellular DC electric fields on excitability in rat hippocampal slices in vitro," J Physiol, vol. 557, pp. 175-90, 2004.

[9] L. Manola, J. Holsheimer, P. Veltink, and J. R. Buitenweg, "Anodal vs cathodal stimulation of motor cortex: a modeling study," Clin Neurophysiol, vol. 118, pp. 46474, 2007.

[10] F. Rattay, "Analysis of the electrical excitation of CNS neurons," IEEE Trans Biomed Eng, vol. 45, pp. 766-72, 1998.

[11] L. Manola, B. H. Roelofsen, J. Holsheimer, E. Marani, and J. Geelen, "Modelling motor cortex stimulation for chronic pain control: electrical potential field, activating functions and responses of simple nerve fibre models," Med Biol Eng Comput, vol. 43, pp. 335-43, 2005.

[12] C. C. McIntyre, W. M. Grill, D. L. Sherman, and N. V. Thakor, "Cellular effects of deep brain stimulation: model-based analysis of activation and inhibition," J Neurophysiol, vol. 91, pp. 1457-69, 2004.

[13] S. K. Esser, S. L. Hill, and G. Tononi, "Modeling the effects of transcranial magnetic stimulation on cortical circuits," J Neurophysiol, vol. 94, pp. 622-39, 2005.

[14] W. S. Anderson, P. Kudela, J. Cho, G. K. Bergey, and P. J. Franaszczuk, "Studies of stimulus parameters for seizure disruption using neural network simulations," Biol Cybern, vol. 97, pp. 173-94, 2007.

[15] R. Leal-Campanario, J. M. Delgado-Garcia, and A. Gruart, "Microstimulation of the somatosensory cortex can substitute for vibrissa stimulation during Pavlovian conditioning," Proc Natl Acad Sci U S A, vol. 103, pp. 10052-7, 2006.

[16] M. Girgis and W. Shih-Chang, A new stereotaxic atlas of the rabbit brain. St. Louis, MO: Warren H. Green Inc, 1981. 
[17] L. Ingber and P. L. Nunez, "Neocortical dynamics at multiple scales: EEG standing waves, statistical mechanics, and physical analogs," Math Biosci, vol. 229, pp. 160-73, 2010 .

[18] W. Gerstner and W. M. Kistler, Spiking Neuron Models : Single Neurons, Populations, Plasticity: Cambridge University Press, 2002.

[19] G. Deco, V. K. Jirsa, P. A. Robinson, M. Breakspear, and K. Friston, "The dynamic brain: from spiking neurons to neural masses and cortical fields," PLoS Comput Biol, vol. 4, pp. e1000092, 2008.

[20] B. Molaee-Ardekani, P. Benquet, F. Bartolomei, and F. Wendling, "Computational modeling of high-frequency oscillations at the onset of neocortical partial seizures: From 'altered structure' to 'dysfunction'," Neuroimage, vol. 52, pp. 1109-1122, 2010.

[21] H. A. Swadlow, "Thalamocortical control of feed-forward inhibition in awake somatosensory 'barrel' cortex," Philos Trans R Soc Lond B Biol Sci, vol. 357, pp. 1717-27, 2002.

[22] H. A. Swadlow, "Fast-spike interneurons and feedforward inhibition in awake sensory neocortex," Cereb Cortex, vol. 13, pp. 25-32, 2003.

[23] I. Bojak and D. T. Liley, "Modeling the effects of anesthesia on the electroencephalogram," Phys Rev E Stat Nonlin Soft Matter Phys, vol. 71, pp. 041902, 2005.

[24] H. R. Wilson and J. D. Cowan, "Excitatory and inhibitory interactions in localized populations of model neurons," Biophys J, vol. 12, pp. 1-24, 1972.

[25] F. Lopes da Silva, "Electrical potentials," in Encyclopedia of the human brain, vol. 2, V. S. Ramachandran, Ed. New York: Academic Press, 2002, pp. 147-67.

[26] F. Frohlich and D. A. McCormick, "Endogenous electric fields may guide neocortical network activity," Neuron, vol. 67, pp. 129-43, 2010.

[27] H. Markram, M. Toledo-Rodriguez, Y. Wang, A. Gupta, G. Silberberg, and C. Wu, "Interneurons of the neocortical inhibitory system," Nat Rev Neurosci, vol. 5, pp. 793807, 2004.

[28] L. G. Nowak, M. V. Sanchez-Vives, and D. A. McCormick, "Lack of orientation and direction selectivity in a subgroup of fast-spiking inhibitory interneurons: cellular and synaptic mechanisms and comparison with other electrophysiological cell types," Cereb Cortex, vol. 18, pp. 1058-78, 2008.

[29] B. Ahmed, J. C. Anderson, K. A. Martin, and J. C. Nelson, "Map of the synapses onto layer 4 basket cells of the primary visual cortex of the cat," J Comp Neurol, vol. 380, pp. 230-42, 1997.

[30] R. Azouz, C. M. Gray, L. G. Nowak, and D. A. McCormick, "Physiological properties of inhibitory interneurons in cat striate cortex," Cereb Cortex, vol. 7, pp. 534-45, 1997.

[31] W. Paulus, "Outlasting excitability shifts induced by direct current stimulation of the human brain," Suppl Clin Neurophysiol, vol. 57, pp. 708-14, 2004.

[32] D. Reato, A. Rahman, M. Bikson, and L. C. Parra, "Low-intensity electrical stimulation affects network dynamics by modulating population rate and spike timing," $J$ Neurosci, vol. 30, pp. 15067-79, 2010.

[33] S. Ozen, A. Sirota, M. A. Belluscio, C. A. Anastassiou, E. Stark, C. Koch, and G. Buzsaki, "Transcranial electric stimulation entrains cortical neuronal populations in rats," J Neurosci, vol. 30, pp. 11476-85.

[34] B. Fritsch, J. Reis, K. Martinowich, H. M. Schambra, Y. Ji, L. G. Cohen, and B. Lu, "Direct current stimulation promotes BDNF-dependent synaptic plasticity: potential implications for motor learning," Neuron, vol. 66, pp. 198-204, 2010.

[35] J. G. Jefferys, "Influence of electric fields on the excitability of granule cells in guineapig hippocampal slices," J Physiol, vol. 319, pp. 143-52, 1981. 
[36] T. Radman, R. L. Ramos, J. C. Brumberg, and M. Bikson, "Role of cortical cell type and morphology in subthreshold and suprathreshold uniform electric field stimulation in vitro," Brain Stimul, vol. 2, pp. 215-28, 228 e1-3, 2009.

[37] K. Matsunaga, M. A. Nitsche, S. Tsuji, and J. C. Rothwell, "Effect of transcranial DC sensorimotor cortex stimulation on somatosensory evoked potentials in humans," Clin Neurophysiol, vol. 115, pp. 456-60, 2004.

[38] A. Dieckhofer, T. D. Waberski, M. Nitsche, W. Paulus, H. Buchner, and R. Gobbele, "Transcranial direct current stimulation applied over the somatosensory cortex differential effect on low and high frequency SEPs," Clin Neurophysiol, vol. 117, pp. 2221-7, 2006.

[39] J. W. Kim and P. A. Robinson, "Compact dynamical model of brain activity," Phys Rev E Stat Nonlin Soft Matter Phys, vol. 75, pp. 031907, 2007.

[40] M. L. Steyn-Ross, D. A. Steyn-Ross, J. W. Sleigh, M. T. Wilson, and L. C. Wilcocks, "Proposed mechanism for learning and memory erasure in a white-noise-driven sleeping cortex," Phys Rev E Stat Nonlin Soft Matter Phys, vol. 72, pp. 061910, 2005.

[41] B. Molaee-Ardekani, L. Senhadji, M. B. Shamsollahi, B. Vosoughi-Vahdat, and E. Wodey, "Brain activity modeling in general anesthesia: enhancing local mean-field models using a slow adaptive firing rate," Phys Rev E Stat Nonlin Soft Matter Phys, vol. 76, pp. 041911, 2007.

[42] B. L. Foster, I. Bojak, and D. T. Liley, "Population based models of cortical drug response: insights from anaesthesia," Cogn Neurodyn, vol. 2, pp. 283-96, 2008.

[43] F. Wendling, "Computational models of epileptic activity: a bridge between observation and pathophysiological interpretation," Expert Rev Neurother, vol. 8, pp. 889-96, 2008.

[44] W. W. Lytton, A. Destexhe, and T. J. Sejnowski, "Control of slow oscillations in the thalamocortical neuron: a computer model," Neuroscience, vol. 70, pp. 673-84, 1996.

[45] O. David, L. Harrison, and K. J. Friston, "Modelling event-related responses in the brain," Neuroimage, vol. 25, pp. 756-70, 2005.

[46] A. C. Marreiros, S. J. Kiebel, and K. J. Friston, "A dynamic causal model study of neuronal population dynamics," Neuroimage, vol. 51, pp. 91-101, 2010.

[47] P. Suffczynski, S. Kalitzin, F. L. da Silva, J. Parra, D. Velis, and F. Wendling, "Active paradigms of seizure anticipation: computer model evidence for necessity of stimulation," Phys Rev E Stat Nonlin Soft Matter Phys, vol. 78, pp. 051917, 2008.

[48] V. Khatri, J. A. Hartings, and D. J. Simons, "Adaptation in thalamic barreloid and cortical barrel neurons to periodic whisker deflections varying in frequency and velocity," J Neurophysiol, vol. 92, pp. 3244-54, 2004.

[49] Z. W. Zhang and M. Deschenes, "Intracortical axonal projections of lamina VI cells of the primary somatosensory cortex in the rat: a single-cell labeling study," J Neurosci, vol. 17, pp. 6365-79, 1997.

[50] A. Gupta, Y. Wang, and H. Markram, "Organizing principles for a diversity of GABAergic interneurons and synapses in the neocortex," Science, vol. 287, pp. 273-8, 2000.

[51] J. J. Ekstrand, M. E. Domroese, S. L. Feig, K. R. Illig, and L. B. Haberly, "Immunocytochemical analysis of basket cells in rat piriform cortex," J Comp Neurol, vol. 434, pp. 308-28, 2001. 
Fig. 1: (A) The structure of the model and the synaptic connections between the subpopulations in the model including principal cells population (type $P$ ) and two types of interneuron populations (type I and type I'). (B) Internal components of a subpopulation (here, type $P$ subpopulation). At the input level of type $P$ subpopulation three pulse-to-wave functions (mimicking the PSPs induced by AMPA, GABA $A_{A, \text { fast }}$ and $\mathrm{GABA}_{\mathrm{A}, \text { slow }}$ receptor activation) provide EPSP and IPSP (fast and slow) components of the mean membrane potential of the pyramidal cells population. A sigmoid wave-to-pulse function transforms the mean membrane potential to a mean firing rate.

Fig. 2 : Coupling model between a subpopulation of neurons in the neural mass model and the electric field resulting from the externally-applied stimulation (tDCS). (A) The variation of the average membrane potential of a given subpopulation is proportional to the intensity of the component of the electric field oriented along the main axis of the cells $\left(\mathrm{E}_{\mathrm{y}}\right)$. A field aligned with the orthodromic direction (dendritic tuft to axon), will result in a positive (depolarizing or excitatory) perturbation of the soma membrane potential. The opposite effect is observed on the dendritic tuft. (B) Schematic diagram showing a given subpopulation of cells, its input pulse-to-wave functions, output wave-to-pulse function, and the polarization effect of the electric field on soma membrane potential.

Fig. 3: (A) Some typical evoked potentials in response to air-puffs (left) under control condition where no tDCS current is applied, and averaged somatosensory evoked potential $(n=10)$ (right). (B, C) The same quantities as in (A) under anodal and cathodal tDCS conditions, respectively. (D) Superposition of evoked potentials in control condition (green) as well as in anodal (red) and cathodal (dark blue) tDCS conditions. Color-coded patches show the interval between $15 \%$ to $85 \%$ percentiles of evoked potential values for each tDCS condition. 
Fig. 4 : (A) Typical simulated EP under control condition (no tDCS effect in the model). The simulated EP includes the most dominant peaks $(N 1 a, N 1 b, P 1, N 2, P 2)$ observed in real aSEP data with correct polarities and latencies. (B) Time course of average EPSP (red), fast IPSP (green) and slow IPSP (blue) components which are summed up at the level of pyramidal cells to approximate the LFP. (C) Parameter sensitivity analysis. Random changes of the parameter vector $\boldsymbol{\theta}=\left\{A, B, G, n_{P}, n_{I}, n_{I^{\prime}}\right\}$ (manually found) lead to changes in the shape of the simulated EP. When parameters stay in the range of $\left[\boldsymbol{\theta}_{0} \pm \alpha . \boldsymbol{\theta}_{0}\right]$ with $\alpha<0.5$, the general shape is conserved.

Fig. 5 : A representative simulated EP under control condition (green) and under cathodal (blue) and anodal (red) tDCS stimulation for each of the four empirically identified EP response classes (panels (A) to (D) correspond to classes 1 to 4 , respectively). Here, tDCS is only applied on the pyramidal cell subpopulation (situation 1): none of these classes fit with actually observed modifications of peak amplitudes and latencies measured in the real SEP.

Fig. 6 : Variation of the amplitude of N1a (panel A), N1b (panel B), P1 (panel C), and N2 (panel D) peaks induced by anodal (left panels) and by cathodal (right panels) tDCS. Under anodal (resp. cathodal) tDCS, pyramidal cells population is always depolarized (resp. hyperpolarized). Interneurons are subjected to be depolarized (indicated by the symbol "D") or hyperpolarized (indicated by the symbol " $\mathrm{H}$ "). Arrows indicate how polarization of interneurons must evolve for the constraints on "features" to be met in the model.

Fig. 7 : Variation of the latencies of N1a (panel A), N1b (panel B), P1 (panel C), and N2 (panel D) peaks induced by anodal (left panels) and by cathodal (right panels) tDCS. 
Fig. 8 : Cost function maps obtained for a different polarization directions of mean membrane potential of type I and type I' interneurons under anodal and cathodal tDCS. Arrows indicate optimum polarization directions for both interneurons.

Fig. 9: Simulated EPs under control (green), anodal (light red), and cathodal (dark blue) tDCS. Here, tDCS effect affects all subpopulations (situation 2): simulated EPs do reproduce actually observed modifications of peak amplitudes and latencies, as measured in the real SEP. In this case, model parameters were set to the numerical values given in table 1 and the relative effect of tDCS on type I and type I' population w.r.t. type $P$ population was $35 \%$ and $50 \%$, respectively.

Table 1: Parameter values of the model corresponding to a typical EP response under the control condition.

Table 2: A summary of the influence of anodal and cathodal tDCS on amplitudes and latencies of main peaks in the EP for each of the four empirically identified classes (in situation 1 where only pyramidal cells are affected by tDCS). In the table "Yes"/"No" stands for the ability/disability of the classes to reproduce a feature correctly as in reality. 
Table 1:

\begin{tabular}{|l|l|}
\hline PSP amplitudes & $A=1.25, B=1.5, G=2 m V$ \\
\hline PSP rate & $a_{1}=50, a_{2}=200 \mathrm{~s}^{-1}$ \\
constants & $b_{1}=40, b_{2}=100 \mathrm{~s}^{-1}$ \\
& $g_{1}=100, g_{2}=350 \mathrm{~s}^{-1}$ \\
\hline Connectivity & $C_{P P}=200, C_{P I}=200, C_{P I^{\prime}}=200$, \\
parameters & $C_{I P}=50, C_{I I}=140$, \\
& $C_{I^{\prime} P}=28, C_{I^{\prime} I}=110, C_{I^{\prime}}=100$ \\
\hline Wilson-Cowan & $Q_{P}^{\max }=50, Q_{I}^{\max }=50, Q_{I}^{\max }=50 \mathrm{~s}^{-1}$ \\
sigmoids & $\theta_{P}=11, \theta_{I}=1.5, \theta_{I^{\prime}}=2 \mathrm{mV}^{-1}$ \\
& $r_{P}=1, r_{I}=1, r_{I^{\prime}}=1.5 \mathrm{mV}^{-1}$ \\
\hline Subcortical & $m_{P}=80, m_{I}=90, m_{I^{\prime}}=60 \mathrm{~s}^{-1}$ \\
Inputs & $n_{P}=200, n_{I}=480, n_{I^{\prime}}=220 \mathrm{~s}^{-1}$ \\
parameters & $\tau=1000 \mathrm{~s}^{-1}$ \\
\hline
\end{tabular}


Table 2:

\begin{tabular}{|c|c|c|c|c|c|}
\hline \multirow{2}{*}{\multicolumn{2}{|c|}{ Classes of model parameters }} & \multirow{3}{*}{ Class 1} & \multirow{3}{*}{ Class 2} & \multirow{3}{*}{ Class 3} & \multirow{3}{*}{ Class 4} \\
\hline & & & & & \\
\hline \multicolumn{2}{|c|}{ Changes observed in the actual aSEP } & & & & \\
\hline \multirow{5}{*}{ Anodal } & increase of N1a amplitude & No & No & No & No \\
\hline & increase of $N 1 b$ amplitude & Yes & Fairly & Yes & Yes \\
\hline & Decrease $P 1$ amplitude & No & No & Yes & Yes \\
\hline & Maintaining $P 1$ latency & Yes & Yes & Yes & Yes \\
\hline & Increase of the frequency of $P 1-N 2-P 2$ complex & No & Yes & Yes & Yes \\
\hline \multirow{5}{*}{ Cathodal } & Decrease of $N 1 a$ & No & No & No & No \\
\hline & Decrease of $N 1 b$ & Yes & Fairly & Yes & Yes \\
\hline & Decrease of $P 1$ amplitude & Yes & Yes & No & No \\
\hline & Increase of $P 1$ latency & No & No & No & No \\
\hline & Merging of $P 1-N 2-P 2$ into a single later peak & No & Fairly & Fairly & Fairly \\
\hline
\end{tabular}


Fig. 1




Fig. 2

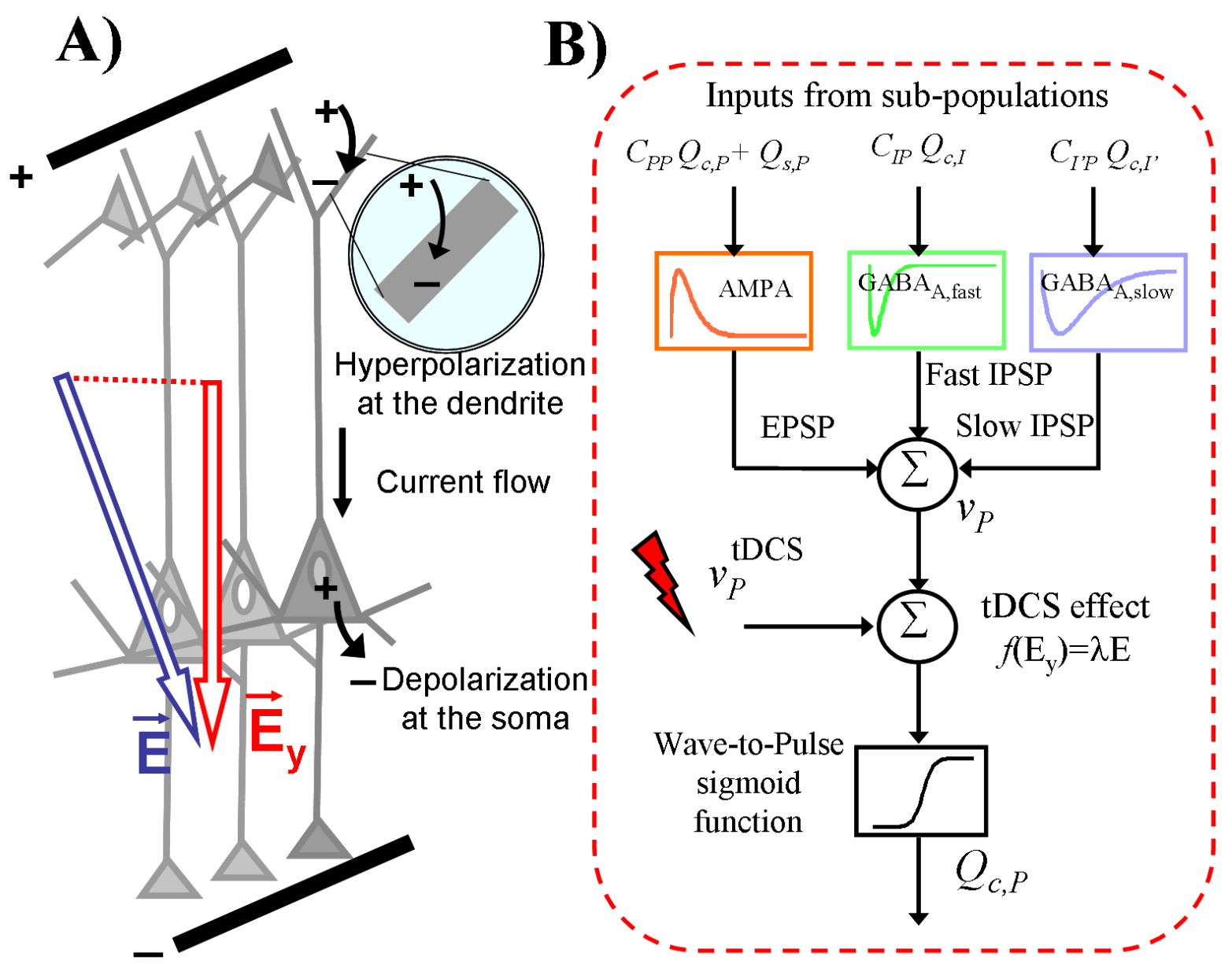


Fig. 3

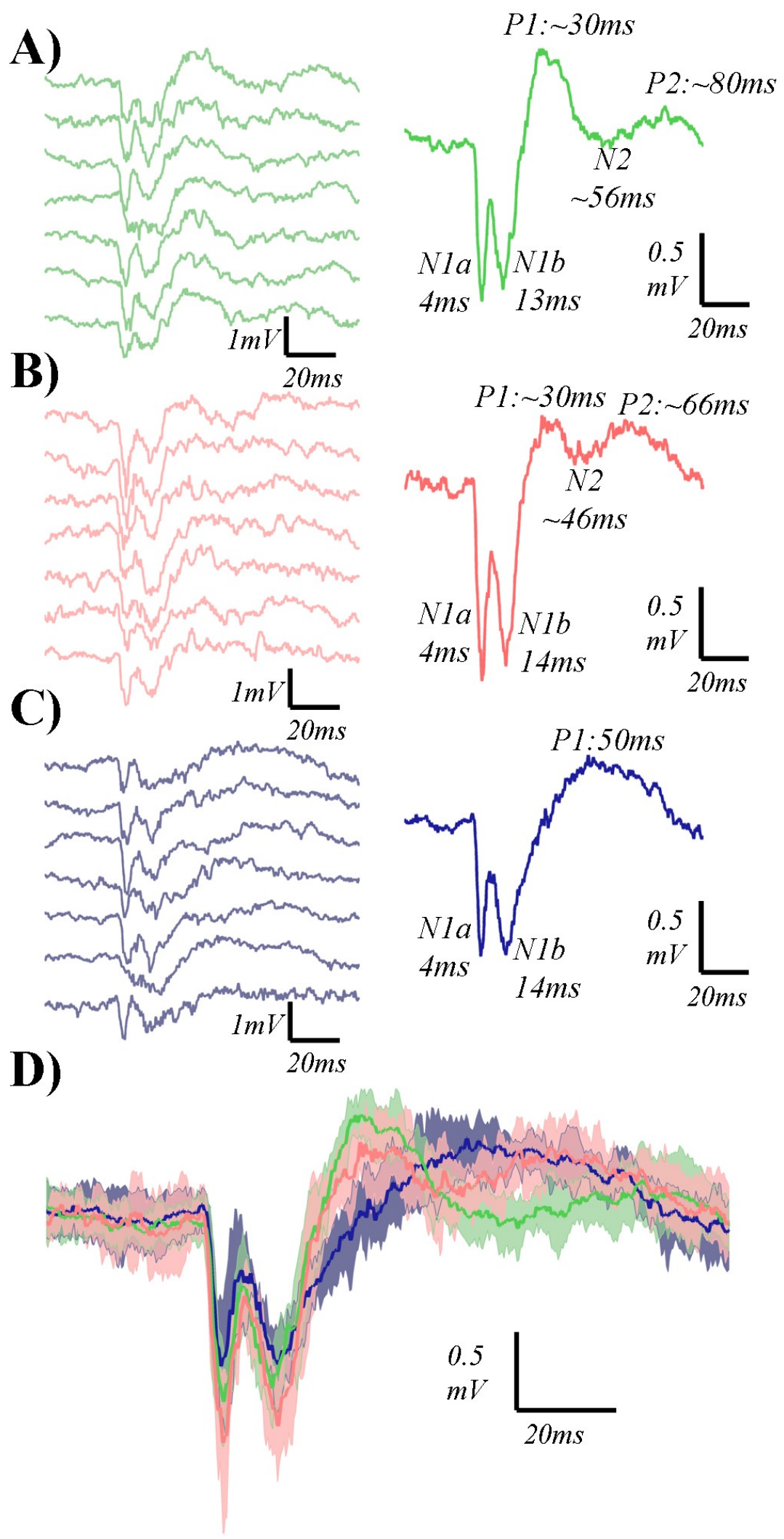


Fig. 4

(A) Simulated EP

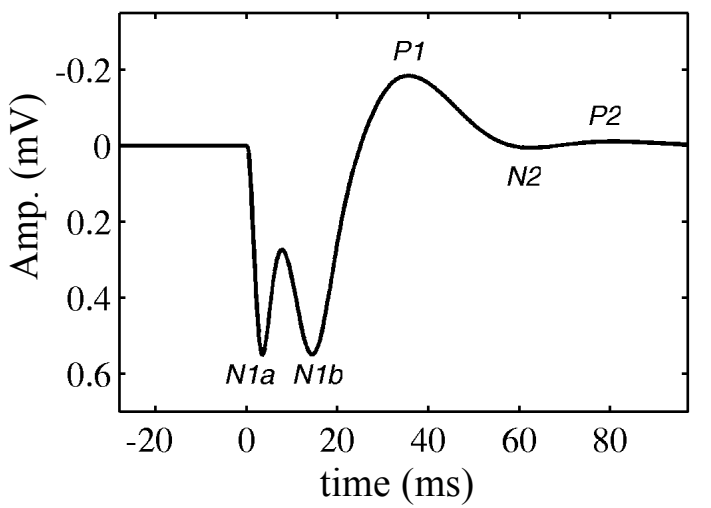

(B) Decomposition of the EP

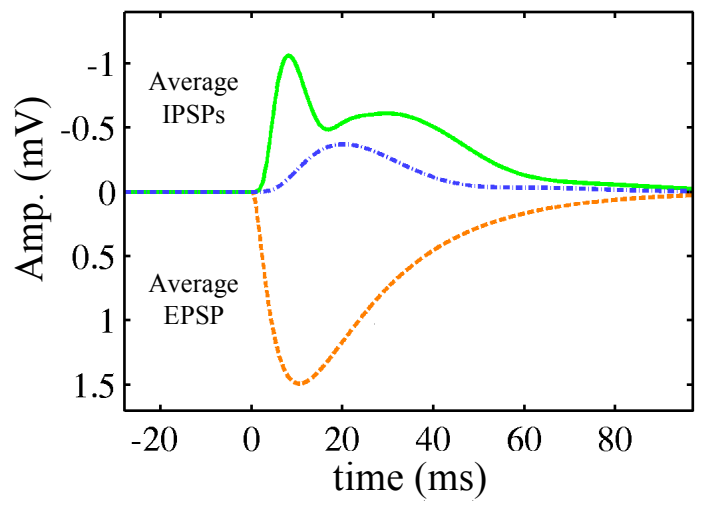

(C) Sensitivity analysis

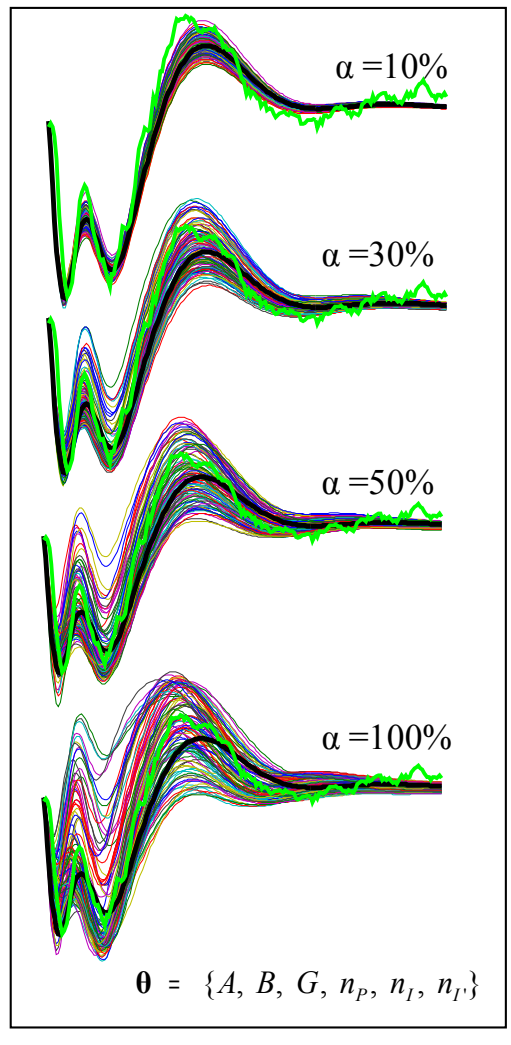


Fig. 5

(A) Class 1

(B) Class 2
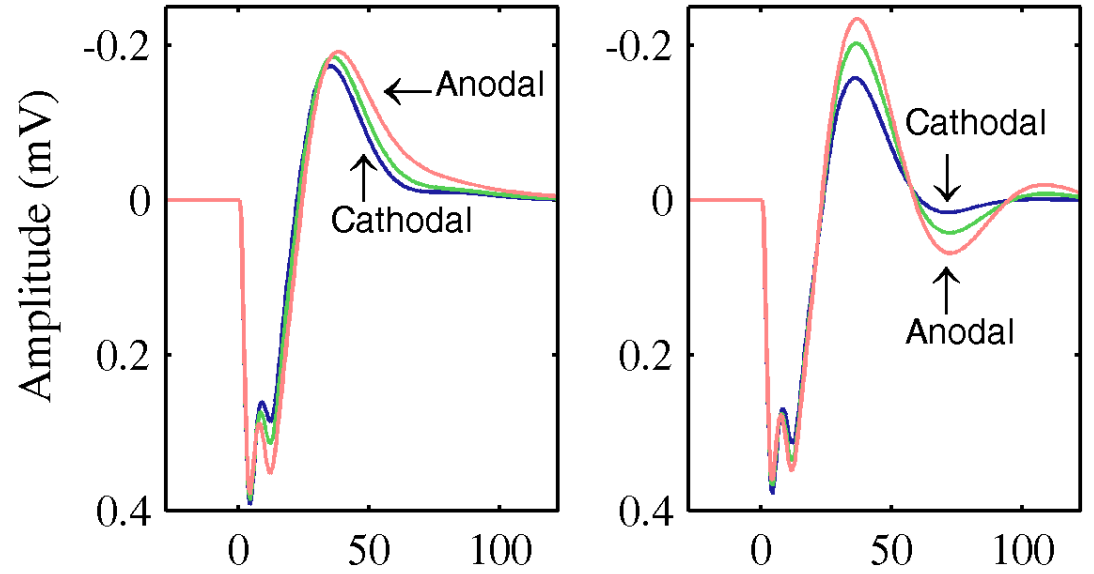

(C) Class 3

(D) Class 4
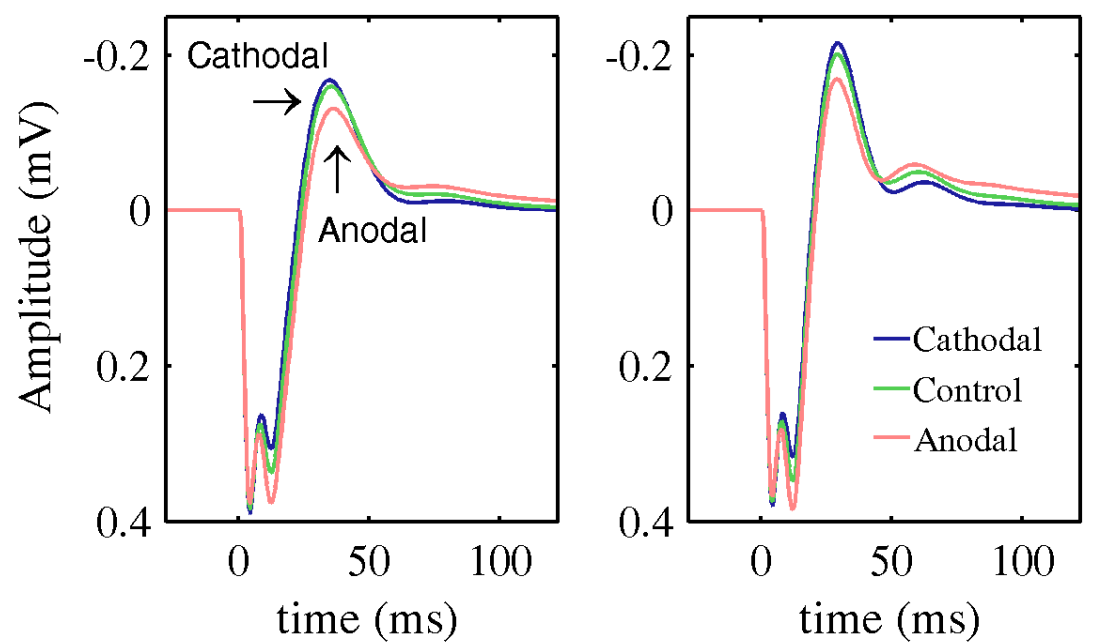
Fig. 6
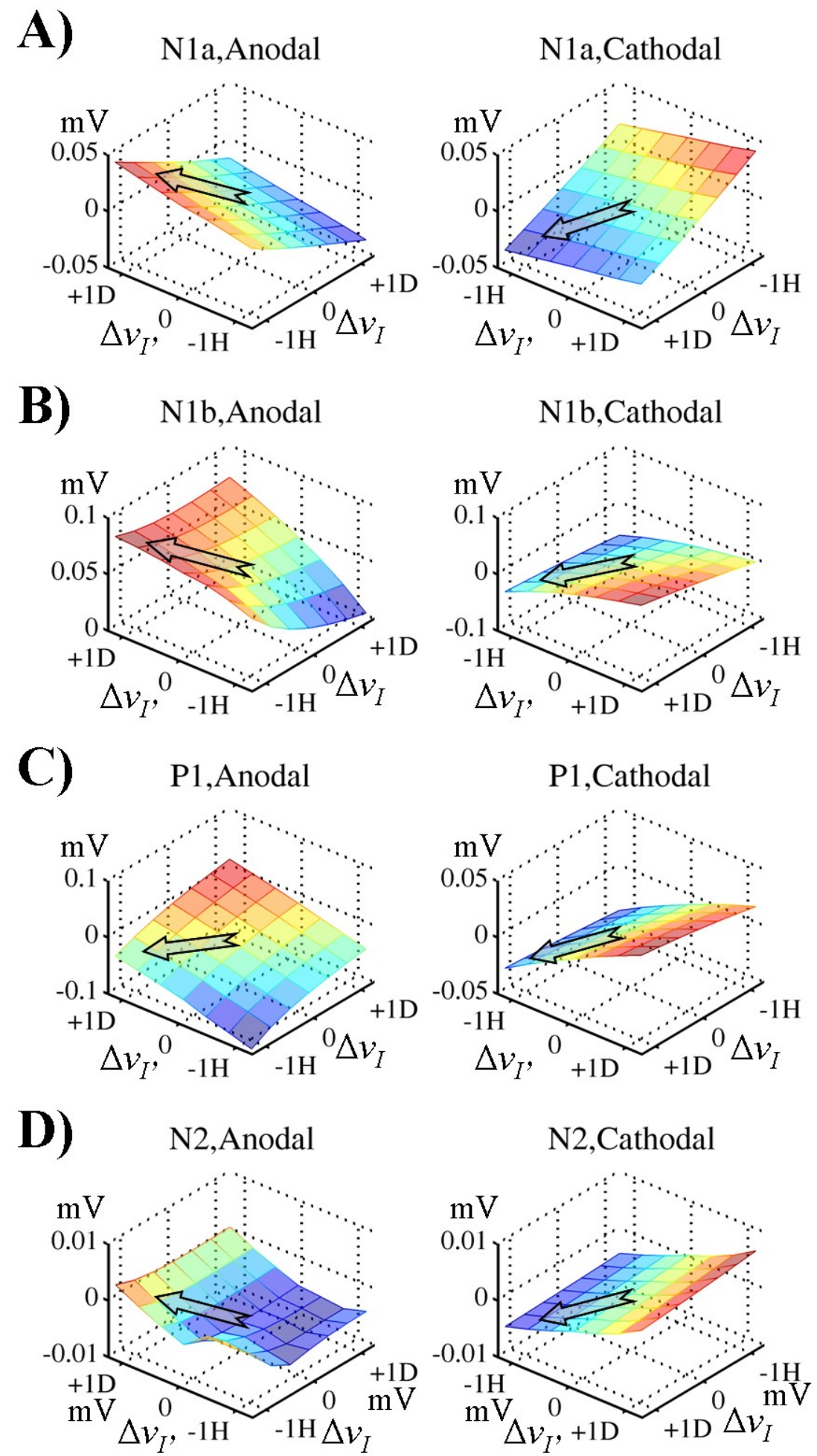
Fig. 7
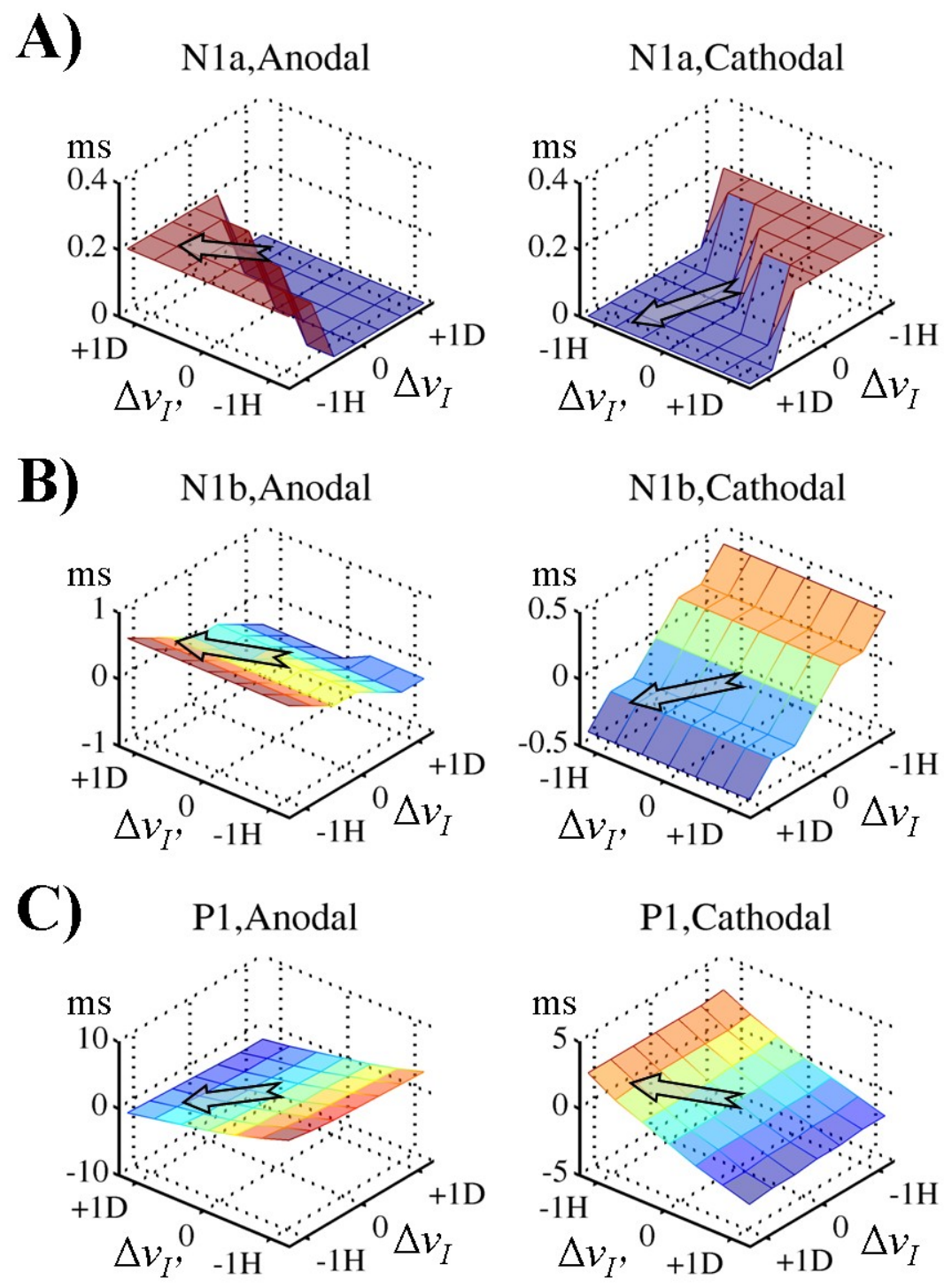

\section{P1,Cathodal}
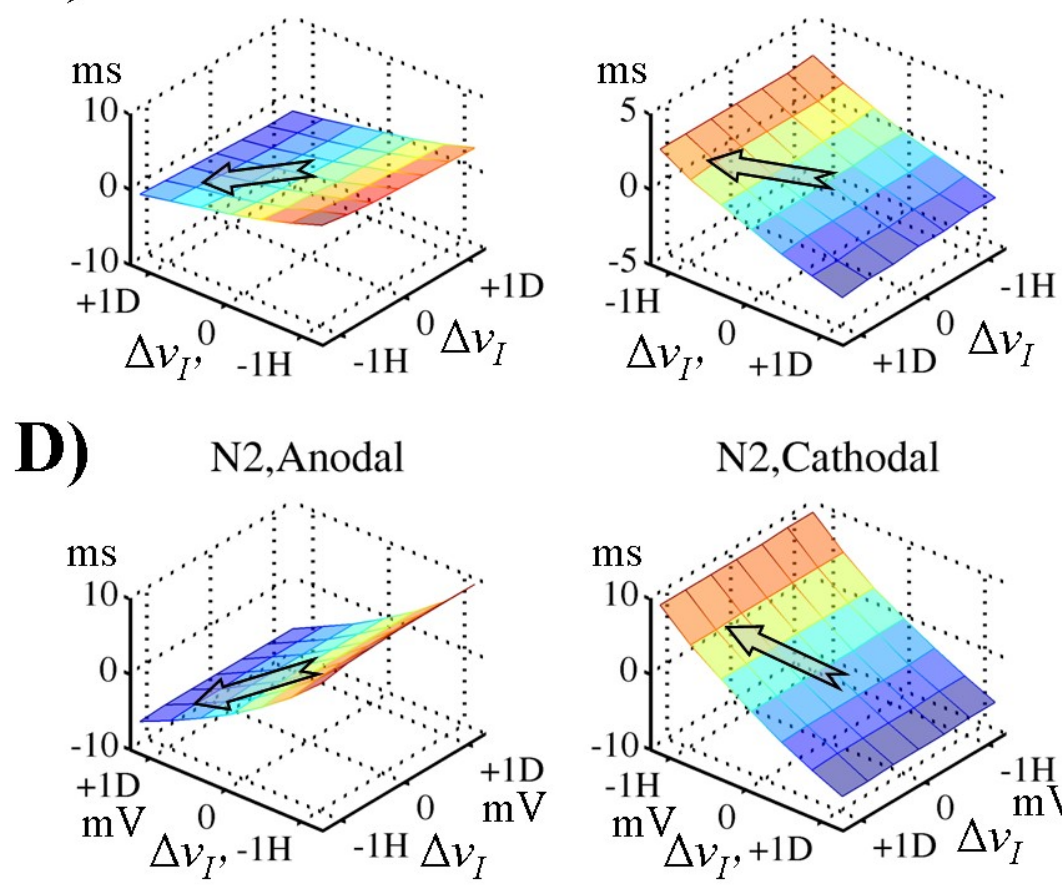

N2,Cathodal

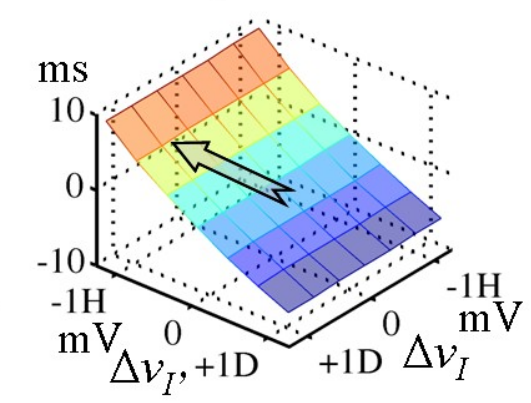


Fig. 8

f, A n o d a 1

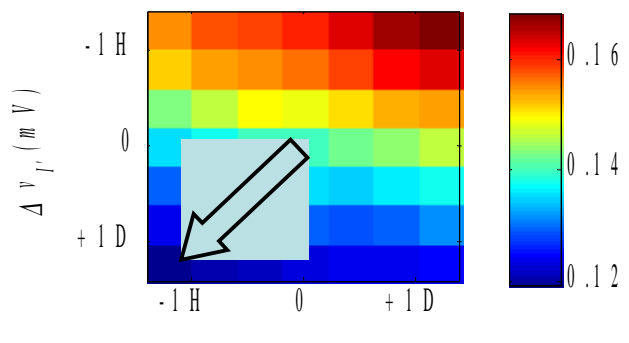

$f$, Cathodal

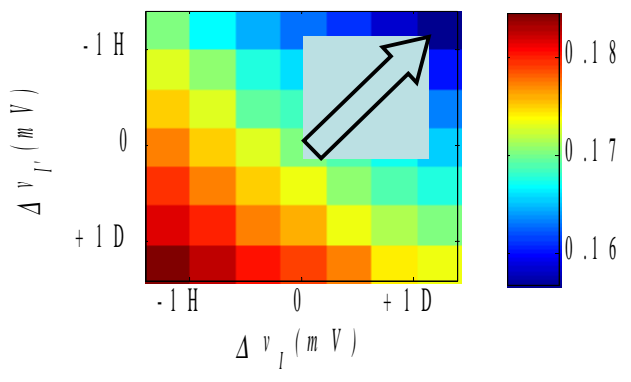


Fig. 9

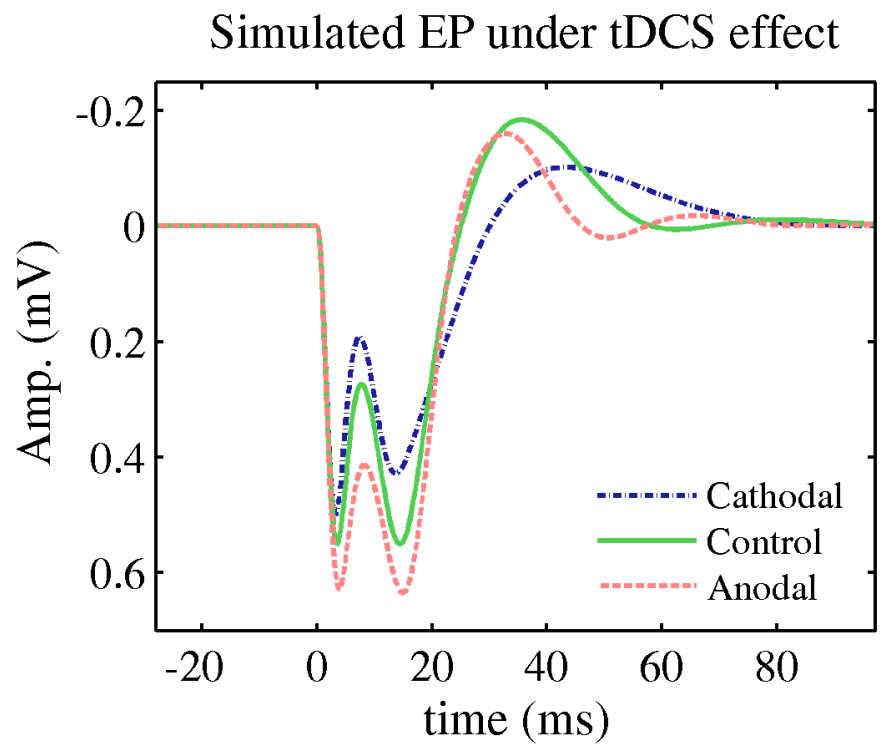

